\title{
Within-host evolution of Enterococcus faecium during longitudinal carriage and transition to bloodstream infection in immunocompromised patients
}

Danesh Moradigaravand ${ }^{1 *+}$, Theodore Gouliouris $2,4,5^{*+}$, Beth Blane ${ }^{2}$, Plamena Naydenova ${ }^{2}$, Catherine Ludden ${ }^{3}$, Charles Crawley ${ }^{4}$, Nicholas M. Brown ${ }^{4,5}$, M. Estée Török ${ }^{2,4,5}$, Julian Parkhill ${ }^{1}$ and Sharon J. Peacock ${ }^{1,2,3}$

\begin{abstract}
Background: Enterococcus faecium is a leading cause of hospital-acquired infection, particularly in the immunocompromised. Here, we use whole genome sequencing of E. faecium to study within-host evolution and the transition from gut carriage to invasive disease.

Methods: We isolated and sequenced 180 E. faecium from four immunocompromised patients who developed bloodstream infection during longitudinal surveillance of E. faecium in stool and their immediate environment.

Results: A phylogenetic tree based on single nucleotide polymorphisms (SNPs) in the core genome of the 180 isolates demonstrated several distinct clones. This was highly concordant with the population structure inferred by Bayesian methods, which contained four main BAPS (Bayesian Analysis of Population Structure) groups. The majority of isolates from each patient resided in a single group, but all four patients also carried minority populations in stool from multiple phylogenetic groups. Bloodstream isolates from each case belonged to a single BAPS group, which differed in all four patients. Analysis of 87 isolates (56 from blood) belonging to a single BAPS group that were cultured from the same patient over 54 days identified 30 SNPs in the core genome (nine intergenic, 13 non-synonymous, eight synonymous), and 250 accessory genes that were variably present. Comparison of these genetic variants in blood isolates versus those from stool or environment did not identify any variants associated with bloodstream infection. The substitution rate for these isolates was estimated to be 128 (95\% confidence interval 79.82 181.77) mutations per genome per year, more than ten times higher than previous estimates for $E$. faecium. Within-patient variation in vancomycin resistance associated with vanA was common and could be explained by plasmid loss, or less often by transposon loss.
\end{abstract}

Conclusions: These findings demonstrate the diversity of E. faecium carriage by individual patients and significant within-host diversity of $E$. faecium, but do not provide evidence for adaptive genetic variation associated with invasion.

Keywords: Enterococcus faecium, Within-host evolution, Genome sequencing

\footnotetext{
* Correspondence: dm16@sanger.ac.uk; theo.gouliouris@doctors.org.uk ${ }^{\dagger}$ Equal contributors

'Wellcome Trust Sanger Institute, Wellcome Genome Campus, Hinxton, Cambridgeshire CB10 1SA, UK

2Department of Medicine, University of Cambridge, Box 157, Addenbrooke's Hospital, Hills Road, Cambridge CB2 OQQ, UK

Full list of author information is available at the end of the article
} 


\section{Background}

Enterococcus faecium is a common gut commensal and a leading cause of nosocomial infection, particularly in the immunocompromised [1]. Bacterial characterisation using multilocus sequence typing (MLST) has demonstrated that hospital-adapted E. faecium are genetically distinct from commensal isolates [2] and cluster within clonal complex (CC) 17, now designated as clade A1 [3]. Isolates belonging to this clade are globally disseminated and have been linked to numerous healthcare-associated outbreaks [4-6]. Their enhanced biological fitness in healthcare settings has been associated with genetic adaptations that alter survival, virulence and antibiotic resistance [1, 3, 7]. Vancomycin-resistant E. faecium (VRE) is of particular concern and limits treatment options $[8,9]$, as recognized by its inclusion in the WHO priority pathogens list for the research and development of new antibiotics [10].

The increasing application of whole genome sequencing (WGS) to clinical settings has begun to elucidate the genomic epidemiology of E. faecium at national and individual hospital levels [11-13]. In addition to confirming the widespread dissemination of hospital-acquired $E$. faecium, these studies have shown that WGS can track VRE transmission during complex healthcare pathways that involve multiple wards within the same hospital [14]. Less is known about the genomic dynamics and evolution of E. faecium within individual patients [15]. This is important as there is growing evidence that short-term within-host evolution is higher than that estimated for long-term evolution and can result in a divergent population [16], which can manifest as variable drug resistance and virulence $[17,18]$. One previous longitudinal study of $E$. faecium carriage by residents of a nursing home described the presence of lineages that varied in vancomycin resistance associated with loss of $v a n A$ in the same individual [19]. Here, we extend these findings through the study of four patients who developed E. faecium bloodstream infection while undergoing longitudinal surveillance of stool carriage, and from whom multiple colonies were sequenced from stool, blood cultures and their environment.

\section{Methods}

\section{Study setting and participants}

Four patients were prospectively identified on two adult haematology wards at the Cambridge University Hospitals NHS Foundation Trust (CUH) between May and Nov 2015 (two cases on each ward) who developed E. faecium bloodstream infection during longitudinal screening for $E$. faecium carriage in stool on admission and weekly until discharge. Blood cultures were taken by the treating doctors following protocols for sepsis investigation. Blood culture sets consisting of three bottles (standard aerobic, anaerobic and FAN, BacT/ALERT, bioMérieux, Marcy
l'Etoile, France) were obtained peripherally and/or centrally. Environmental samples were also obtained from patient bedside and bathroom/toilet areas from three cases on the day of discharge using flocked swabs (FLOQSwabs, Copan Italia spa, Brescia, Italy).

\section{Microbiology}

Blood cultures were processed by the routine diagnostic laboratory. For those positive for E. faecium, up to ten colonies were randomly selected from each positive blood culture set by picking colonies from all positive primary plates onto which bottles were sub-cultured. Environmental swabs were inoculated into Brain Heart Infusion broth, incubated at $37{ }^{\circ} \mathrm{C}$ in air overnight and $100 \mu \mathrm{L}$ sub-cultured onto $\mathrm{BBL}^{\mathrm{TM}}$ Enterococcosel ${ }^{\mathrm{TM}}$ agar (BD, Oxford, UK) supplemented with $30 \mathrm{mg} / \mathrm{L}$ ampicillin (Sigma-Aldrich, Poole, UK) to select for ampicillin-resistant E. faecium), and Brilliance VRE (Oxoid, Basingstoke, UK) to select for vancomycin-resistant E. faecium. A single colony was selected from each environmental sample, with priority given to vancomycin-resistant $E$. faecium if present. Stool samples were cultured onto two solid media (Enterococcosel agar with ampicillin, and Brilliance VRE chromogenic agar) and into two enrichment broths (Enterococcosel (BD, Oxford, UK) and Enterococcosel with $6 \mathrm{mg} / \mathrm{L}$ vancomycin). In addition, the first stool sample for each patient was cultured using media without antibiotic selection (Slanetz-Bartley agar (Oxoid), and Enterococcosel broth). Culture media and broths were incubated at $37{ }^{\circ} \mathrm{C}$ in air for up to $48 \mathrm{~h}$, with the addition of shaking for liquid culture. For broths that turned black (indicative of enterococcal growth), $100 \mu \mathrm{L}$ was sub-cultured onto Enterococcosel agar with/ without ampicillin or Brilliance VRE, respectively. Up to five colonies were picked from each plate for confirmation of species using matrix-assisted laser desorption/ionization time-of-flight mass spectrometry (MALDI-TOF MS; Biotyper version 3.1, Bruker Daltonics, Coventry, UK). Phenotypic antimicrobial susceptibility testing was determined using the P607 card on the Vitek 2 instrument (version 7.01, bioMérieux, Marcy l'Étoile, France). Categorization into susceptible, intermediate and resistant was done according to EUCAST clinical breakpoints (http://www.eucast.org/clinical_breakpoints/) except for high-level gentamicin and highlevel streptomycin resistance, which were validated against CLSI synergy testing (500 and $1000 \mathrm{mg} / \mathrm{L}$, respectively).

\section{Sequencing and population genomics analysis}

DNA was extracted and DNA libraries prepared and sequenced on an Illumina HiSeq2000 with 100-cycle pairedend runs as described previously [20] to give an average depth of 85-fold. MLST sequence types (STs) were derived from Illumina read data using the script available at https:// github.com/sanger-pathogens/mlst_check. A Velvet-based algorithm [21] was used to produce de novo multi-contig 
assemblies, which were annotated with Prokka [22], and the output used to reconstruct the pan-genome using Roary [23]. A core genome was created for each isolate using a $99 \%$ cut-off, with a default $95 \%$ identity cut-off. Scoary [24] was used with 50 re-samplings in the permutation test to find significant associations between the presence and absence of genes in the pan-genome and antibiotic susceptible/resistant and carriage/disease phenotypes. Single nucleotide polymorphisms (SNPs) were extracted from the core-genome alignment produced by Roary using the script available at https://github.com/sanger-pathogens/snp-sites. Sites with ambiguous calls were removed and the output used to construct a neighbour-joining phylogenetic tree with the ape and phangorn packages in $\mathrm{R}$. The tree and associated metadata were visualised using iTOL [25] and Figtree (http://tree.bio.ed.ac.uk/software/figtree/).

\section{Population structure and phylogenetic Bayesian analysis}

We used the core genome alignment produced by Roary and extracted the SNPs with an in-house tool, available at https://github.com/sanger-pathogens/snp-sites. We then used the SNP presence or absence as the input for Scoary to identify variants strongly associated with the source of isolation. The SNP alignment was also used to estimate the genetic population structure by hierBAPS [26], which is a nested clustering approach, with five, seven and ten values for estimated number of clusters and two iterations. After identifying four BAPS clusters that contained isolates associated with bloodstream infection (referred to here as BAPS 1, 2, 3 and 4), a Bayesian phylogenetic tree was obtained for isolates within each group so as to obtain a more accurate picture of recent evolution within each BAPS group. To achieve this, the reads were first mapped to a nominated reference sequence in each cluster based on the best assembly statistics (largest N50 value). SMALT v0.7.4 (http://www.sanger.ac.uk/science/tools/smalt-0) was then used on each cluster with the maximum and minimum insert sizes of 1000 and 50, respectively. SAMtools mpileup and BCFtools were used to detect SNPs, with the parameter values of a minimum base quality of 50 and minimum root mean squared mapping of 30 to identify SNPs. SNPs at sites in which the variant was present in $<75 \%$ of paired-end reads were excluded. Mapping to a reference genome can be inaccurate if the reference genome has a different structure based on genomic re-arrangements or other genetic variation. However, in our analysis the local reference was highly similar to the other isolates within the same BAPS group ( $>99 \%$ identity) and is unlikely to have a significantly different genomic architecture.

We then used Gubbins [27] with five iterations to remove high SNP density regions, which are indicative of recombination. To assess the temporal signal for each cluster, the root-to-tip distance values obtained by constructing a neighbour-joining tree was plotted against time (day) of isolation, using 10,000 bootstraps with re-sampled days to attain a distribution for R-squared values. The significance of the real R-squared was evaluated based on the distribution.

BEAST v1.7 [28] was then used on the SNP alignment to obtain the Bayesian tree. Isolates were assigned based on the sample from which each was cultured (blood culture, stool or environmental sampling), which were defined as a discrete "source" trait and then used to reconstruct the counts of state change, as detailed in [29]. BEAST was run using date of isolation and various models that included a strict molecular clock model with uniform and lognormal distribution for base frequency and a lognormal distribution for the transition of location status. Three independent MCMC chains were run for 50 million generations (sampling every 1000 generations). The convergence was confirmed if the effective sample size exceeded 200, after excluding the first $10 \%$ states as burn-in. The TreeAnnotator tool, which is part of the BEAST package, was then used to obtain confidence intervals for the node ages. The key parameters, including tree root height and clock rate, of different runs from different models were in agreement.

Using the alignment from the Bayesian analysis, the SeqTrack function in the Adegenet $\mathrm{R}$ package was used to construct a network for within-host isolates for each BAPS cluster. This reconstructs the most parsimonious network structure based on pairwise SNP distances and date of isolation $[30,31]$. The results were visualized using functions in the igraph package in $\mathrm{R}$.

The marginal likelihood values for the ancestral state for presence/absence of plasmids were estimated with the rerootingMethod function (the SYM function) in the phytools $\mathrm{R}$ package. The results were then displayed with the plotTree function.

\section{Identification of virulence factors, antibiotic resistance and plasmids}

Virulence factors, resistance genes and plasmid replicons were identified using the srst2 package [32] using 90\% coverage and $10 \%$ divergence cut-offs. Virulence factors were curated by extracting known and putative virulence factors for E. faecium from multiple sources, including the virulence factor database (http://www.mgc.ac.cn/VFs/). The list of virulence factors is available in Additional file 1. The plasmid replicon database and antibiotic resistance genes were downloaded from the srst2 package. The presence of plasmids was further evaluated by reconstructing plasmid assemblies using plasmidSPAdes [33], based on their coverage. After confirming the presence of a plasmid, the genomic context of resistance genes was confirmed by comparing the plasmid containing the gene of interest against the non-redundant nucleotide NCBI database using BLAST. Paired-end reads were then mapped to the 
reference genome of known previously sequenced plasmids using the same approach as above to confirm the presence of the plasmid. The sequence and the annotation files of the plasmid in BAPS2 group, reported in [14], are available in Additional file 2.

\section{Results}

Our analysis was based on the whole genomes of $180 \mathrm{E}$. faecium isolates cultured from the blood and stool of four hospital in-patients (termed patients A-D) and the environment of three of these cases (see Additional file 3: Figure S1 for sampling timeline). To define the population diversity of the entire collection, a phylogenetic tree was constructed based on SNPs in the core genome (Fig. 1a). This demonstrated several distinct clones, which were highly concordant with the population structure inferred by hierBAPS (Fig. 1a). Four different BAPS groups (termed 1-4) contained isolates associated with bloodstream infection (Fig. 1a, c). Although the majority of isolates from each patient resided in a single BAPS group, the four patients also carried minority populations in stool that belonged to multiple BAPS groups, a diversity that was maintained over time (Additional file 3: Figure S2).
Bloodstream isolates from each patient belonged to a single BAPS group that differed in each individual (Fig. 1a, b). BAPS1, BAPS2, BAPS3 and BAPS4 contained blood isolates from patient $\mathrm{A}, \mathrm{B}, \mathrm{C}$ and $\mathrm{D}$, respectively. Bloodstream isolates in some patients were related to stool carriage isolates from other cases (Fig. 1a, b). Specifically, stool isolates from patient $B$ resided in BAPS1 group that contained blood isolates from patient A. Furthermore, another stool isolate from patient B together with an environmental isolate from patient $\mathrm{D}$ resided in the same BAPS group as blood isolates from patient C (Fig. 1b). These findings suggest that isolates from bloodstream infections are closely linked with carriage isolates, which may be shared by different patients.

Multilocus sequence types (STs) were derived from the sequence data for the isolate collection. This revealed multiple STs, the majority of which were ST80 $(n=136)$ with the remainder being ST262 $(n=10)$, ST78 $(n=26)$ and ST280 $(n=4)$, all of which belong to the hospitalassociated clade A1 (CC17), together with two isolates with novel STs (Fig. 1a). To confirm the clade assignment for the study isolates we compared these with the genomes of isolates from A1, A2 and B clade drawn
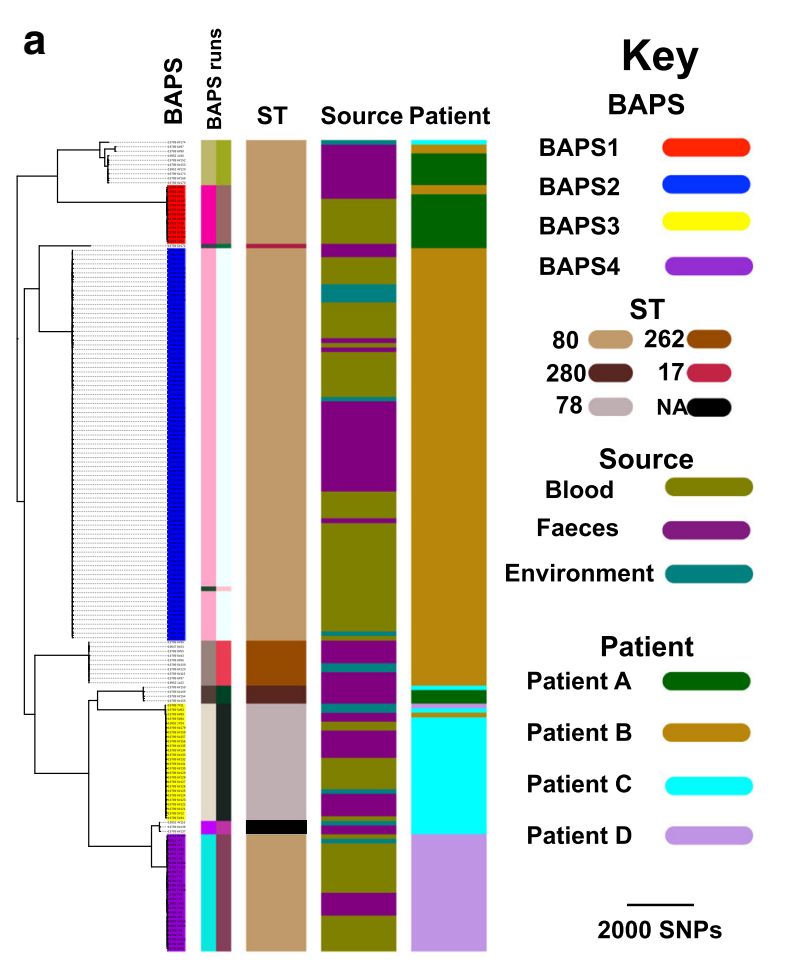

b

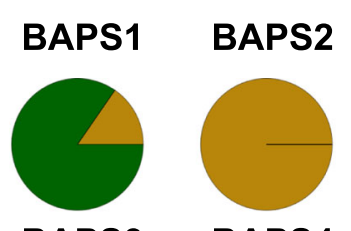

BAPS3 BAPS4

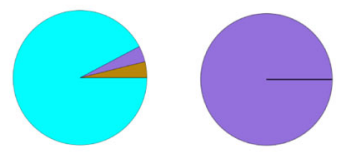

C

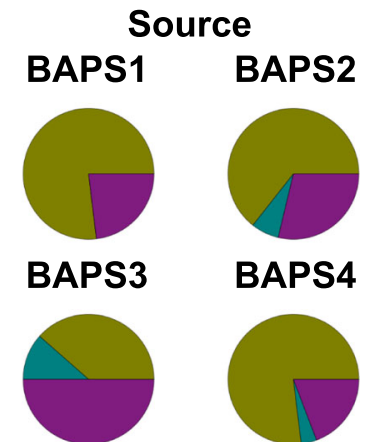

Fig. 1 a Neighbour-joining tree of 180 E. faecium genomes used in this study. BAPS clusters 1, 2, 3 and 4 are inferred BAPS groups that all contained invasive isolates. The pie charts for BAPS groups 1, 2, 3 and 4 contain 13, 87, 26 and 26 isolates, respectively. The BAPS groups were on average 4797.97 SNPs apart. The average pairwise SNP distance for isolates within BAPS1, 2, 3 and 4 were 3.97, 2.13, 2.86 and 1.30, respectively. The BAPS run columns correspond to clustering results with five and ten values for the estimated numbers of clusters in the hierBAPS analysis. Each colour signifies one group. BAPS groups 1,2,3 and 4, which contained invasive isolates, were inferred with both parameter sets. The root of the tree is the midpoint of the two most distant taxa in the collection. Frequency of isolates based on patient (b) and source of isolation (c) across four BAPS groups 
from a diverse collection described previously [3], which were recovered from various ecological environments. The phylogenetic tree of isolates constructed from the core genome confirmed that our isolates resided in clade A1 (Additional file 3: Figure S3). We noted that STs had poor resolution in capturing the within-patient population diversity, with incomplete concordance between the ST tree and whole genome tree (see ST80 clades in Fig. 1a), which can be attributed to recombination in MLST loci. This indicates the superiority of WGS in understanding fine resolution of within-host population structure, as described previously [12, 34].

For the analysis of within-host bacterial diversity, we first examined isolates from patient B who had 102 isolates (56 blood, 38 stool, eight environmental) recovered from 22 specimens (eight blood, six stool, eight environmental) over a period of 54 days, including a period of persistent bacteraemia over 9 days (Additional file 3: Figure S1). Phylogenetic analysis of the 102 isolates demonstrated that most isolates resided in BAPS2 (56 blood, 25 stool, six environmental), and were assigned to ST80, ST262 and ST78, which were each distributed across the tree (Fig. 1). The stool E. faecium population was assigned to BAPS1, BAPS2 and additional minor groups (Additional file 3: Figure S2). Despite the presence of multiple strain types in stool, only ST80/BAPS2 isolates from stool were closely related to isolates cultured from blood (Fig. 1a). These were present in stool before the first episode of bacteraemia, suggesting that the patient developed invasive disease with their carriage isolate (Fig. 1a; Additional file 3: Figure S2). A more detailed analysis of diversity in BAPS2 was undertaken to explore whether one or more genetic features were over-represented in invasive isolates and could be associated with the process of invasion or survival in the bloodstream. This included an analysis of mutations and the presence/absence of accessory genes. SNPs were identified in patient B/BAPS2 isolate genomes by mapping to a relevant reference genome and removing hypervariable recombinant regions (Fig. 2; Additional file 4). This identified 30 SNPs, of which nine were intergenic, 13 were non-synonymous and eight were synonymous. The non-synonymous mutations occurred in regulator proteins, $\mathrm{ABC}$ transporters and putative membrane proteins. A pan-genome analysis identified 250 accessory genes $(\sim 7 \%$ of the pan-genome) that were variable in patient B BAPS2 isolates (Additional file 5). This included genes carried by the plasmid that also carried vancomycin resistance genes (see below). The other genes were mainly insertion elements, phage-related and hypothetical proteins. Comparison of the presence of SNPs or accessory genes in blood versus other isolates, including environmental and stool isolates, did not reveal an association between the presence/absence of either an accessory gene or SNP and blood isolates (Additional file 3: Figure S4). This suggests that invasion was not linked with a particular adaptive variant.

We noted that patient B BAPS2 isolates had a strong temporal signal (R-squared value for the dataset was greater than those of $99 \%$ of replicas in the bootstrap test) and used Bayesian analysis to further explore within-host population diversity over time in this patient (Fig. 2). The substitution rate calculated for within-host E. faecium evolution was 128 (95\% confidence interval (CI) 79.82 181.77) mutations per genome per year, which is more than ten times higher than previous estimates for E. faecium based on a collection of clinical isolates in which each isolate was from a different patient [14]. This discrepancy is consistent with previous suggestions that within-patient substitution rates are higher due to increased genetic drift and limited time for purifying selection to act on mildly deleterious mutations [16]. We repeated the Bayesian analysis for a subset of 20 isolates drawn from the four BAPS groups, i.e. five isolates from each BAPS group, for all four patients and found a lower substitution rate of 18.25 (95\% CI 11.52 45.63) mutations per genome per year, which is closer to previous estimates [14,35]. Due to the low number of variants within the BAPS groups, a comparison between strength of selection within and across lineages was not possible, but we identified a total of 678 variants (120 intergenic, 187 synonymous, 371 non-synonymous) that were fixed in the lineages across BAPS groups (Additional file 6). The non-synonymous variants occurred in different functional groups of genes, including membrane proteins, surface proteins, including transporters and facilitators, and regulatory genes. Whether or not these variants contain any adaptive significance needs verification from experimental functional studies.

The phylogenetic tree of patient B BAPS2 isolates demonstrated some mixing of blood, stool and environmental isolates but was consistent with stool isolates being ancestral to those isolated from blood as well as later isolates from stool and the environment (Fig. 2). Stool to blood transition could also be inferred from a transmission network reconstructed for BAPS2 isolates, which indicated high connectivity between stool, blood and environmental isolates (Fig. 3). In the network, carriage isolates gave rise to multiple blood isolates, some of which were closely linked with other stool and environmental isolates. This is consistent with the possible repeated translocation of E. faecium from gut into blood (Fig. 3). Both the transmission network and the phylogenetic tree included cases where blood isolates appeared to be ancestral to stool and environmental isolates; this is likely to be due to the presence of ancestral stool isolates that were not sampled. 

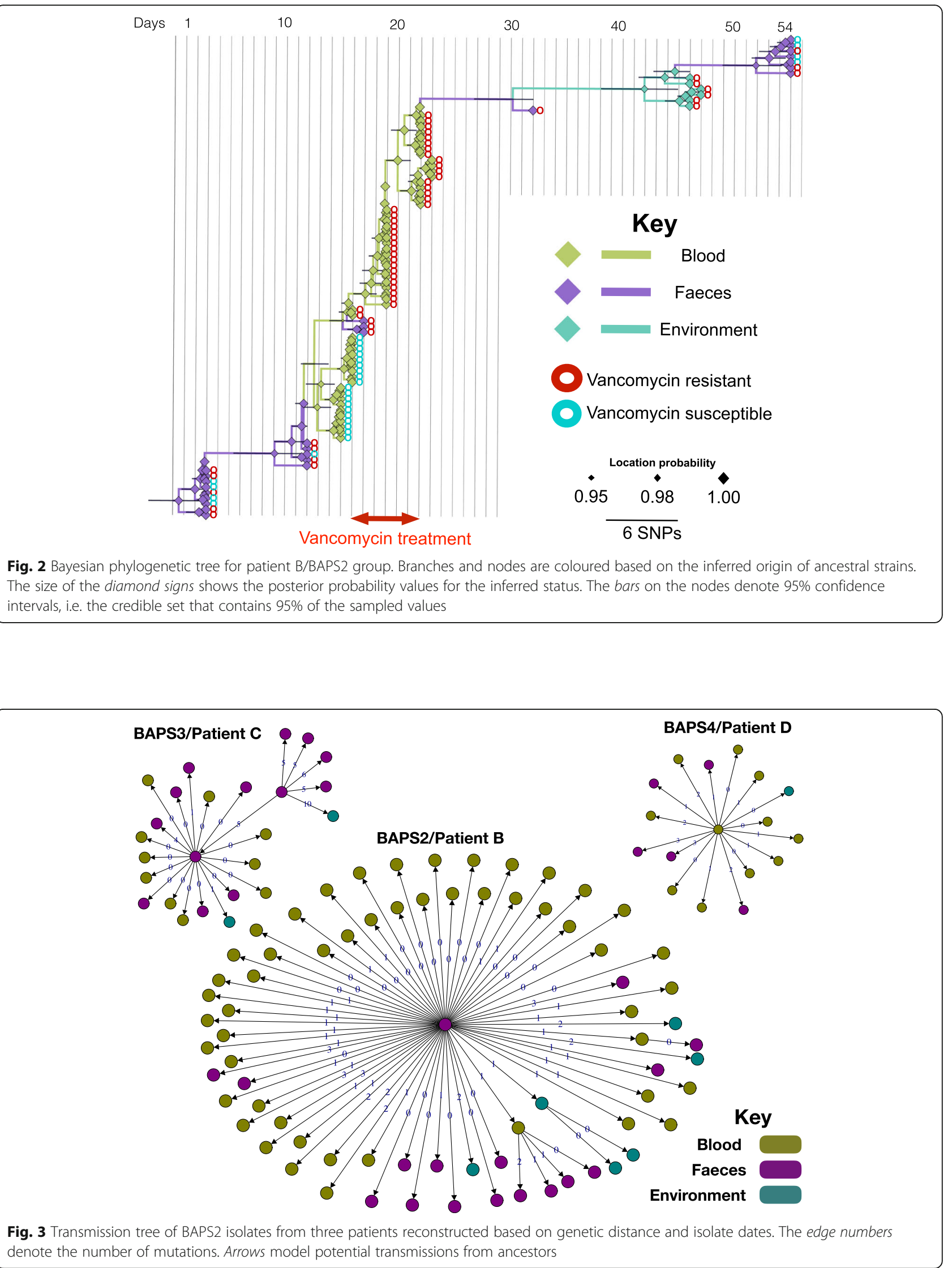
The presence of antibiotic resistance genes was mapped across the isolate collection from all four cases. This revealed a high degree of lineage specificity, with little variation within specific BAPS groups (Fig. 4). Only vancomycin resistance genes and $d f r$ were variably present in closely related blood, stool and environmental isolates in BAPS2 (Fig. 4). Consideration of within-host isolate resistance was focused on the largest subset from patient B, who carried isolates in stool that were variably resistant to streptomycin, gentamicin and quinupristin/ dalfopristin. These resided in different BAPS groups, but BAPS2 blood isolates were universally resistant to streptomycin but susceptible to gentamicin and quinupristin/dalfopristin (Fig. 5). By contrast, patient B BAPS2 isolates were variably resistant to vancomycin and teicoplanin (Fig. 5). To explore this further, we determined the genomic context of vancomycin resistance genes and the presence of transposon Tn1546. This demonstrated that the transposon was carried by a plasmid with the same backbone described previously in E. faecium isolates associated with bacteraemia at the same study hospital [14]. The van genes and associated plasmid were present in ancestral stool isolates but were absent in multiple strains that were recovered later (Additional file 4: Figures S5 and S6). The uncertainty about the status of the ancestral stool isolates, in terms of the presence of the plasmid and the resistance gene, suggests that the population initially contained a mixture of vancomycin susceptible and resistant isolates and that the initial bacteraemia isolates were all susceptible (Additional file 3: Figure S6). Strikingly, under vancomycin treatment, both the stool and the bloodstream population of E. faecium shifted to vancomycin-resistant strains. Thirty-six days after vancomycin treatment was discontinued, a susceptible population of E. faecium was again detectable in stool. The findings suggest a dynamic balance between vancomycinresistant and susceptible E. faecium sub-populations where plasmid or van genes can be lost in some stool isolates in the absence of antibiotic treatment, but the resistant subpopulation is maintained and selected under antibiotic pressure (Additional file 3: Figure S6).

Analysis of within-host diversity in the three other cases revealed several similarities and some differences compared with patient $\mathrm{B}$. For instance, patient $\mathrm{A}$ isolates $(n=22$ : ten blood, 12 stool) had stool isolates that resided in three different clades (Fig. 1a). A single stool isolate in BAPS1 was genetically mixed with, and was on average 2.5 SNPs different from, the blood isolates (Additional file 3: Figure S7). Blood and stool isolates from patient $\mathrm{A}$ were also related to stool isolates from patient $B$, which were ancestral and had an average SNP distance of $\sim 116$ SNPs. All patient A blood isolates were vancomycin resistant due to vanA carried by the plasmid identified in patient $B$ isolates (results not shown). We identified 221 accessory genes that were

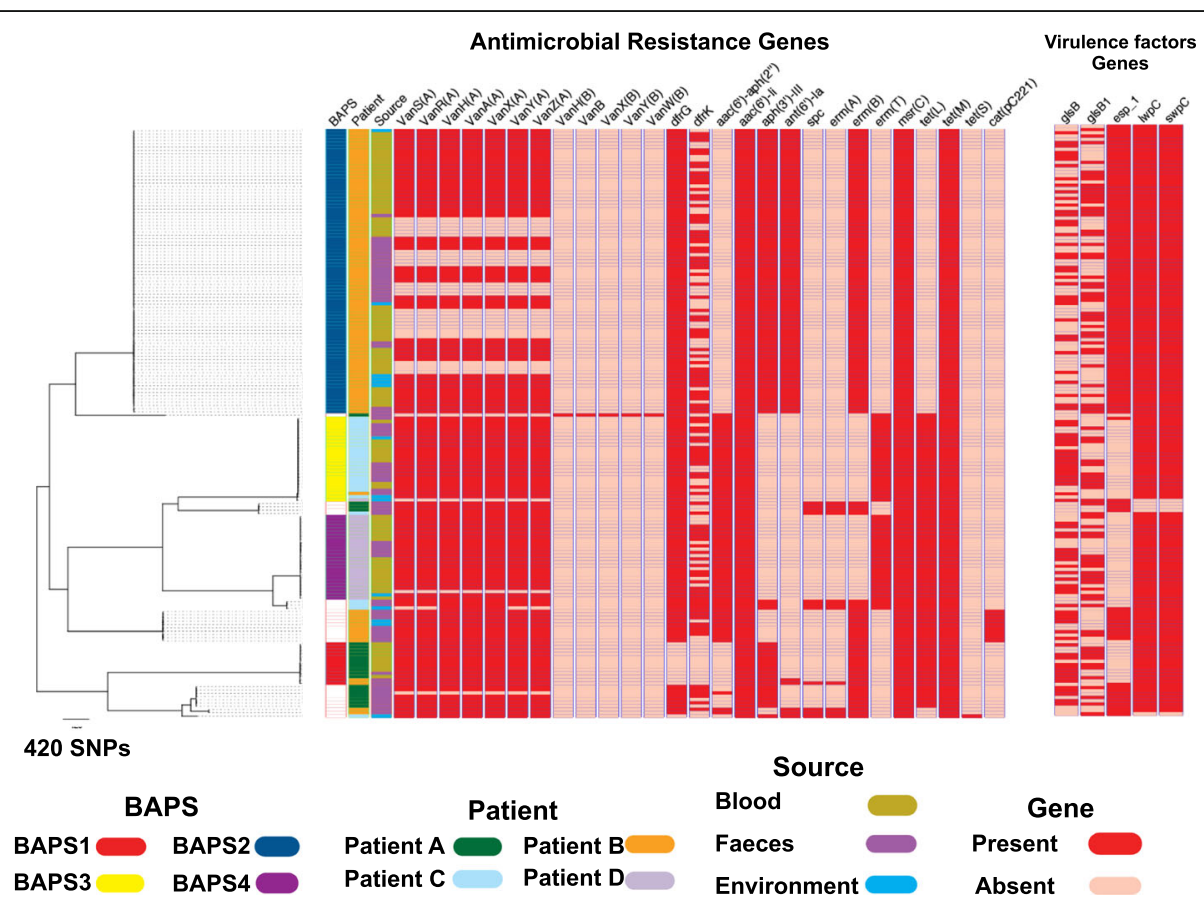

Fig. 4 Phylogenetic distribution of genes encoding antibiotic resistance and virulence factors. Note that only virulence factors that were variably present in the population are shown, meaning that some of the well-known virulence factors, e.g. sagA and at/AEfm, present in every isolate are not shown. The sequence file for the virulence factors studied here is provided in Additional file 1. Note that the vanB-containing isolate was phenotypically susceptible to vancomycin due to lack of $\operatorname{vanh}_{B}$ and $v a n S_{B}$ 


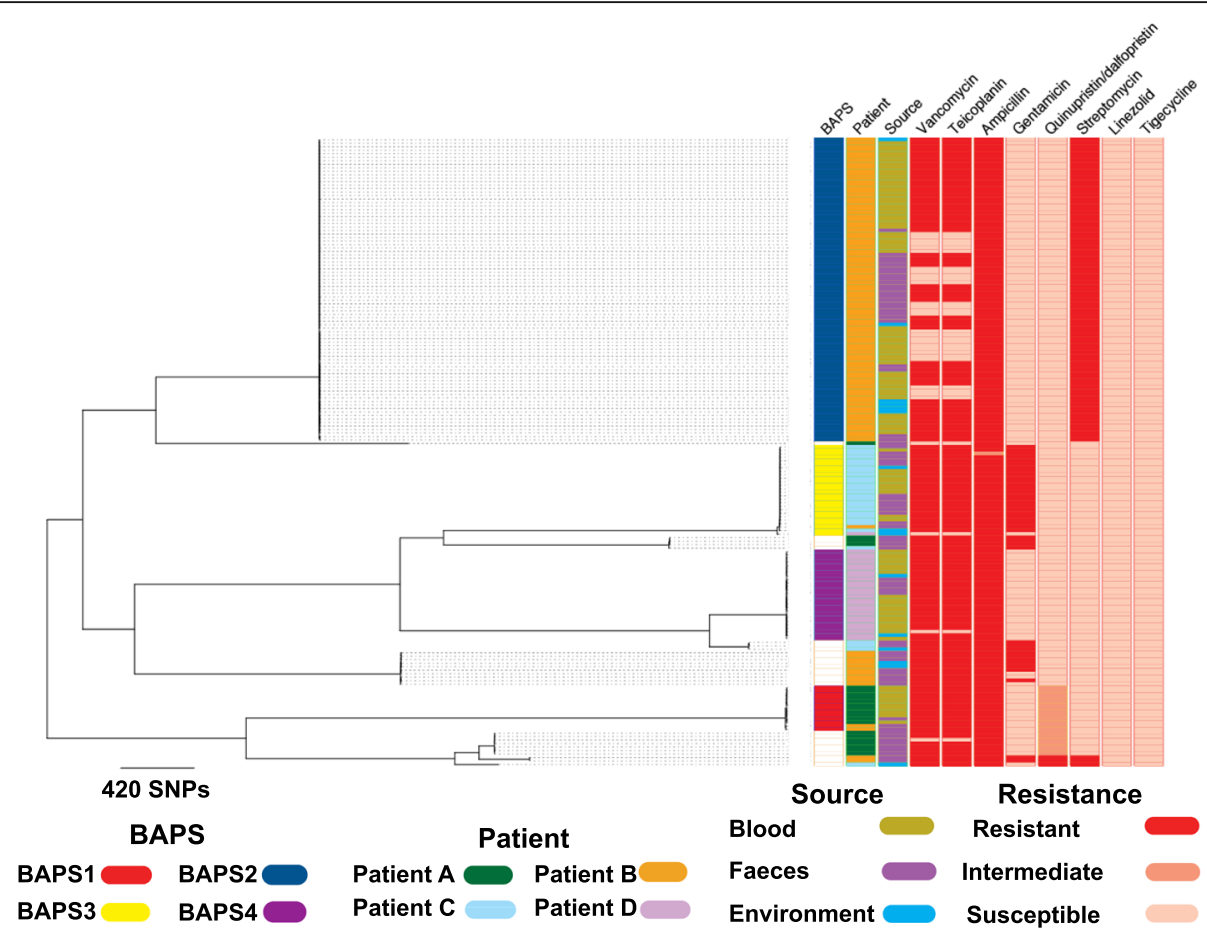

Fig. 5 Frequency of resistant and susceptible isolates across the phylogenetic tree for antibiotics tested in this study

variably present in patient $\mathrm{A}$ isolates within the BAPS1 group. The stool isolate appeared to lack fms 20 encoding a cell surface protein (Additional file 5). To address whether or not the gene confers adaptive importance would require experimental verification, although we note that this gene was not differentially distributed in blood and stool isolates from other patients.

Patient C isolates ( $n=29$ : ten blood, 15 stool, four environment) resided across three clades in the phylogenetic tree (Fig. 1a; Additional file 3: Figures S1 and S2). The majority (24/29) resided in BAPS3, which also contained an isolate from the environment of patient $D$ and a stool isolate from patient $\mathrm{B}$, which were ancestral to those from patient A (Fig. 1b; Additional file 3: Figure S7). In the phylogenetic tree, blood isolates formed a single clade that originated from ancestral stool isolates, and there was an indication of single or multiple introductions of isolates from stool into the environment (Additional file 4: Figure S6), the transmission network confirming that stool isolates served as sources for blood and environmental isolates (Fig. 3). Except for one environmental isolate, the other isolates in BAPS3 from patient A were vancomycin resistant and had a vancomycin resistance transposon inserted into the backbone of the same plasmid as the patient B isolates. A set of 68 accessory genes, mainly mobile genetic elements, were variably present in patient $\mathrm{C}$ isolates within BAPS3 (Additional file 6). In addition, eight non-synonymous, seven synonymous and four intergenic mutations and two indels were identified
(Additional file 5). None of these were associated with source of isolation.

Patient D isolates ( $n=27$ : 20 blood, two environmental, five stool) resided in two BAPS groups. The majority (26) clustered in BAPS4 and differed by nine SNPs (three nonsynonymous, two synonymous and four intergenic), while the remaining isolate resided with isolates from patient $C$ in BAPS3 (Fig. 1; Additional file 3: Figure S7). In contrast to other patients, E. faecium was not recovered from stool obtained 9 and 5 days prior to the onset of bacteraemia but blood isolates clustered with stool and environmental isolates cultured subsequently (Additional file 3: Figure S7). The van genes resided on a different plasmid to the other cases, the plasmid backbone contained a replicon recovered previously from an individual in New York, USA (plasmid accession ID CP018831) [36], our data showing $68 \%$ coverage of that plasmid. One blood isolate was vancomycin susceptible and lacked the whole plasmid, including the resistance gene (Additional file 3: Figure S7). We identified 147 accessory genes, some of which were plasmid related and included vancomycin resistance genes (Additional file 6).

The association between source of isolation and variations in SNPs and accessory genes was also assessed for the isolates from all four patients using Scoary (see "Methods"). However, no variant was identified that was associated with source of isolation.

We screened the collection for specific virulence genes (Additional file 7), the majority of which were present in 
every isolate or were lineage specific and not linked with the origin of isolates (blood versus stool) (Fig. 4). We detected variability in the presence of $g l s$ genes $(g l s A$ and $g l s B)$, which are involved in bile resistance and are crucial for adaptation to the intestinal environment [37], but these were not over-represented in invasive isolates compared with isolates from stool (for example, present in 48 and $52 \%$ of the blood and stool isolates in BAPS2, respectively).

\section{Discussion}

We utilized WGS to characterize E. faecium associated with stool carriage and bloodstream infection in four patients over time. Each patient carried several distinct clades in their stool, but only one of these was isolated from blood in each case. In the overall collection, BAPS groups containing blood isolates also contained stool and environmental isolates, indicating that the diversity of invasive isolates is part of the broader diversity of the carriage population, and that isolates from the immediate environment were derived from carriage. A comparative genomic analysis of related isolates in stool and blood of the same patient failed to reveal adaptive mutations associated with invasive disease. This is consistent with the opportunistic nature of infection in haematooncology patients who have numerous risk factors for systemic infection, including the presence of intravascular and urinary catheters, neutropaenia and loss of mucosal barrier integrity. We found that sub-populations of nearly identical isolates at the core genome differed in the presence of van genes with evidence of loss of the van transposon and/or the plasmid, suggesting instability. However, the maintenance of a resistant sub-population is crucial for its survival under antibiotic pressure and can result in a rapid shift towards its dominance in both the stool and the bloodstream.

We estimated a higher substitution rate for withinhost isolates than has previously been calculated for $E$. faecium from population studies. A higher substitution rate for within-patient diversity is not limited to $E$. faecium and has been reported for other major pathogens such as Helicobacter pylori and methicillin-resistant Staphylococcus aureus (MRSA), and attributed to factors such as increased drift within patients, enhanced by population compartmentalization, repeated selective sweeps and fluctuation in population size, along with limited time for removal of mildly deleterious mutations [16].

The lack of adaptive genetic changes associated with the carriage-invasive transition contrasts with the within-host analysis of other pathogens such as MRSA, where the transition of carriage to invasive status has been linked with the emergence of multiple adaptive mutations that result in truncated proteins [18]. Several explanations are possible for the lack of evidence for adaptive changes allowing invasion in E. faecium. Our study patients were receiving treatment for haematological malignancy and so were immunocompromised, had reduced integrity of mucosal barriers, intravenous devices and repeated exposure to antibiotics. Such circumstances are associated with colonization by drugresistant bacteria, and translocation of bacteria from the gut into the bloodstream [38]. It is also the case that hospital-adapted E. faecium belonging to $\mathrm{CC} 17$ have acquired a genetic repertoire associated with persistence and nosocomial infection, a pre-adaption that may be sufficient to facilitate invasive disease [2, 39]. Although our study systematically studied E. faecium recovered from the stool and blood of patients over time, our cases were restricted to patients who were immunocompromised, and only a small number were studied. This is a potential limitation of the study, and invasive disease in people who are immunocompetent may still be associated with specific adaptive bacterial changes, which could be discovered with a larger sample size. A larger dataset and more comprehensive screening are required to determine whether the findings described here can be more widely generalized.

Our study adds to previous evidence for the carriage of numerous $E$. faecium lineages by hospitalized patients, and the value of using genome sequencing to detect this [14]. This means that picking a single bacterial colony from a sample for sequencing to determine the genetics of E. faecium carriage is likely to capture the dominant clade but will overlook minor lineages, which nonetheless could be associated with transmission and environmental contamination. We found evidence for considerable sharing of highly related isolates between stool and the environment of the same patient, which is consistent with environmental contamination. We also found sharing of highly related isolates between the four cases, which extends findings from a previous study in the same hospital of $E$. faecium transmission associated with $E$. faecium bloodstream infection [14].

\section{Conclusions}

Sequencing of numerous E. faecium isolates from four patients over a period during which they developed bloodstream infection has indicated that opportunistic infection is not associated with bacterial adaptation for the transition from carriage to the invasive disease state.

\section{Additional files}

Additional file 1: Sequence file (fasta) containing list of virulence factors studied here. (TXT $60 \mathrm{~kb}$ )

Additional file 2: The sequence and annotation files of the plasmid, containing vancomycin resistance genes, in the BAPS2 group. (ZIP $95 \mathrm{~kb}$ ) Additional file 3: Supplementary figures. (PDF 920 kb) 
Additional file 4: List of SNPs identified within the BAPS groups for each patient. (CSV $10 \mathrm{~kb}$ )

Additional file 5: List of accessory genes identified within the BAPS groups for each patient. (CSV $31 \mathrm{~kb}$ )

Additional file 6: List of SNPs identified across lineages. (CSV 109 kb)

Additional file 7: List of virulence factors and their corresponding publication and evidence. VFDB refers to the virulence factor database. (CSV $2 \mathrm{~kb}$ )

Additional file 8: List of accession numbers and metadata for isolates. (CSV $56 \mathrm{~kb}$ )

\section{Acknowledgements}

The authors thank the Wellcome Trust Sanger Institute library construction, sequence and core informatics teams. We gratefully acknowledge the contribution of the nurses and healthcare workers on the Haematology wards at Cambridge University Hospitals NHS Trust for assistance with sample collection and the support of the ward matrons, Ann Green and Claire Cowling. We also thank Lois Chaparadza and Dr Rosie Swayne for assistance with sample and clinical data collection. The flocked swabs were kindly donated by Copan Italia spa.

\section{Funding}

This publication presents independent research supported by the Health Innovation Challenge Fund (WT098600, HICF-T5-342), a parallel funding partnership between the Department of Health and Wellcome Trust. The views expressed in this publication are those of the author(s) and not necessarily those of the Department of Health or Wellcome Trust. This project was also funded by a grant awarded to the Wellcome Trust Sanger Institute (098051). TG is a Wellcome Trust Research Training Fellow (103387/Z/13/Z). CL is a Wellcome Trust Sir Henry Postdoctoral Fellow (110243/Z/15/Z).

\section{Availability of data and materials}

All sequences have been submitted to the European Nucleotide Archive (ENA) under the study number PRJEB12937 (https://www.ebi.ac.uk/ena/data/view/ PRJEB12937), and individual accession numbers are given in Additional file 8

\section{Authors' contributions}

TG, SJP and JP designed the study. BB, PN, CL, CC, NMB and MET conducted experimental parts of the study. DM designed, implemented and conducted the majority of data analysis. DM and TG were responsible for the analysis. DM, TG, SJP and JP interpreted the results. DM, TG and SJP wrote the manuscript. JP and SJP were responsible for the management of the study. All authors read and approved the final manuscript.

\section{Ethics approval and consent to participate}

The study was approved by the CUH Research and Development Department (reference A093285) and the National Research Ethics Service East of England Ethics Committee (reference 14/EE/1123). Written informed consent was obtained from all participants prior to enrolment to the study. All human subjects were adult. We declare that the study conformed to the Declaration of Helsinki.

\section{Consent for publication}

Not applicable.

\section{Competing interests}

The authors declare that they have no competing interests.

\section{Publisher's Note}

Springer Nature remains neutral with regard to jurisdictional claims in published maps and institutional affiliations.

\section{Author details}

${ }^{1}$ Wellcome Trust Sanger Institute, Wellcome Genome Campus, Hinxton, Cambridgeshire CB10 1SA, UK. ²Department of Medicine, University of Cambridge, Box 157, Addenbrooke's Hospital, Hills Road, Cambridge CB2 OQQ, UK. ${ }^{3}$ London School of Hygiene and Tropical Medicine, London WC1E 7HT, UK. ${ }^{4}$ Cambridge University Hospitals NHS Foundation Trust,
Addenbrooke's Hospital, Hills Road, Cambridge CB2 OQQ, UK. ${ }^{5}$ Public Health England, Clinical Microbiology and Public Health Laboratory, Cambridge University Hospitals NHS Foundation Trust, Cambridge, UK.

Received: 6 September 2017 Accepted: 7 December 2017

Published online: 27 December 2017

\section{References}

1. Arias CA, Murray BE. The rise of the Enterococcus: beyond vancomycin resistance. Nat Rev Microbiol. 2012;10(4):266-78. doi:10.1038/nrmicro2761. PubMed PMID: 22421879; PubMed Central PMCID: PMCPMC3621121.

2. Willems RJ, Top J, van Santen M, Robinson DA, Coque TM, Baquero F, et al. Global spread of vancomycin-resistant Enterococcus faecium from distinct nosocomial genetic complex. Emerg Infect Dis. 2005;11(6):821-8. doi:10. 3201/eid1106.041204. PubMed PMID: 15963275; PubMed Central PMCID: PMCPMC3367597.

3. Lebreton F, van Schaik W, McGuire AM, Godfrey P, Griggs A, Mazumdar V, et al. Emergence of epidemic multidrug-resistant Enterococcus faecium from animal and commensal strains. MBio. 2013;4(4). doi: 10.1128/mBio.00534-13. PubMed PMID: 23963180; PubMed Central PMCID: PMCPMC3747589.

4. McCracken M, Wong A, Mitchell R, Gravel D, Conly J, Embil J, et al. Molecular epidemiology of vancomycin-resistant enterococcal bacteraemia: results from the Canadian Nosocomial Infection Surveillance Program, 19992009. J Antimicrob Chemother. 2013;68(7):1505-9. doi:10.1093/jac/dkt054.

5. Tedim AP, Ruiz-Garbajosa P, Rodriguez MC, Rodriguez-Banos M, Lanza VF, Derdoy $L$, et al. Long-term clonal dynamics of Enterococcus faecium strains causing bloodstream infections (1995-2015) in Spain. J Antimicrob Chemother. 2017;72(1):48-55. doi:10.1093/jac/dkw366.

6. Palazzo IC, Pitondo-Silva A, Levy CE, da Costa Darini AL. Changes in vancomycin-resistant Enterococcus faecium causing outbreaks in Brazil. J Hosp Infect. 2011;79(1):70-4. doi:10.1016/j.jhin.2011.04.016.

7. Guzman Prieto AM, van Schaik W, Rogers MR, Coque TM, Baquero F, Corander J, et al. Global emergence and dissemination of Enterococci as nosocomial pathogens: attack of the clones? Front Microbiol. 2016;7:788. doi:10.3389/fmicb.2016.00788. PubMed PMID: 27303380; PubMed Central PMCID: PMCPMC4880559.

8. Uttley $\mathrm{AH}$, Collins $\mathrm{CH}$, Naidoo J, George RC. Vancomycin-resistant enterococci. Lancet. 1988;1(8575-6):57-8.

9. Cheah AL, Spelman T, Liew D, Peel T, Howden BP, Spelman D, et al. Enterococcal bacteraemia: factors influencing mortality, length of stay and costs of hospitalization. Clin Microbiol Infect. 2013;19(4):E181-9. doi:10.1111/ 1469-0691.12132.

10. World Health Organization. Global priority list of antibiotic-resistant bacteria to guide research, discovery, and development of new antibiotics. 2017.

11. Pinholt M, Gumpert $H$, Bayliss S, Nielsen JB, Vorobieva V, Pedersen M, et al. Genomic analysis of 495 vancomycin-resistant Enterococcus faecium reveals broad dissemination of a vanA plasmid in more than 19 clones from Copenhagen, Denmark. J Antimicrob Chemother. 2017;72(1):40-7. doi:10. 1093/jac/dkw360.

12. van Hal SJ, Ip CL, Ansari MA, Wilson DJ, Espedido BA, Jensen SO, et al. Evolutionary dynamics of Enterococcus faecium reveals complex genomic relationships between isolates with independent emergence of vancomycin resistance. Microb Genom. 2016;2(1). doi: 10.1099/mgen.0.000048. PubMed PMID: 27713836; PubMed Central PMCID: PMCPMC5049587.

13. Howden BP, Holt KE, Lam MM, Seemann T, Ballard S, Coombs GW, et al Genomic insights to control the emergence of vancomycin-resistant enterococci. MBio. 2013;4(4). doi: 10.1128/mBio.00412-13. PubMed PMID: 23943759; PubMed Central PMCID: PMCPMC3747580.

14. Raven KE, Gouliouris T, Brodrick H, Coll F, Brown NM, Reynolds R, et al. Complex routes of nosocomial vancomycin-resistant enterococcus faecium transmission revealed by genome sequencing. Clin Infect Dis. 2017:64(7): 886-93. doi:10.1093/cid/ciw872.

15. Reuter S, Ellington MJ, Cartwright EJ, Koser CU, Torok ME, Gouliouris T, et al. Rapid bacterial whole-genome sequencing to enhance diagnostic and public health microbiology. JAMA Intern Med. 2013;173(15):1397-404. doi: 10.1001/jamainternmed.2013.7734. PubMed PMID: 23857503; PubMed Central PMCID: PMCPMC4001082

16. Didelot X, Walker AS, Peto TE, Crook DW, Wilson DJ. Within-host evolution of bacterial pathogens. Nat Rev Microbiol. 2016;14(3):150-62. doi:10.1038/ nrmicro.2015.13. PubMed PMID: 26806595; PubMed Central PMCID: PMCPMC5053366. 
17. Kennemann L, Didelot X, Aebischer T, Kuhn S, Drescher B, Droege M, et al. Helicobacter pylori genome evolution during human infection. Proc Natl Acad Sci U S A. 2011;108(12):5033-8. doi:10.1073/pnas.1018444108. PubMed PMID: 21383187; PubMed Central PMCID: PMCPMC3064335.

18. Young BC, Golubchik T, Batty EM, Fung R, Larner-Svensson H, Votintseva AA, et al. Evolutionary dynamics of Staphylococcus aureus during progression from carriage to disease. Proc Natl Acad Sci U S A. 2012;109(12):4550-5. doi: 10.1073/pnas.1113219109. PubMed PMID: 22393007; PubMed Central PMCID: PMCPMC3311376.

19. Brodrick HJ, Raven KE, Harrison EM, Blane B, Reuter S, Torok ME, et al. Whole-genome sequencing reveals transmission of vancomycin-resistant Enterococcus faecium in a healthcare network. Genome Med. 2016;8(1):4. doi:10.1186/s13073-015-0259-7. PubMed PMID: 26759031; PubMed Central PMCID: PMCPMC4709893.

20. Moradigaravand D, Reuter S, Martin V, Peacock SJ, Parkhill J. The dissemination of multidrug-resistant Enterobacter cloacae throughout the UK and Ireland. Nat Microbiol. 2016;1:16173. doi:10.1038/nmicrobiol.2016.173.

21. Zerbino DR, Birney E. Velvet: algorithms for de novo short read assembly using de Bruijn graphs. Genome Res. 2008;18(5):821-9. doi:10.1101/gr.074492.107.

22. Seemann T. Prokka: rapid prokaryotic genome annotation. Bioinformatics. 2014;30(14):2068-9. doi:10.1093/bioinformatics/btu153. PubMed PMID: WOS: 000339814300017

23. Page AJ, Cummins CA, Hunt M, Wong VK, Reuter S, Holden MT, et al. Roary: rapid large-scale prokaryote pan genome analysis. Bioinformatics. 2015; 31(22):3691-3. doi:10.1093/bioinformatics/btv421.

24. Brynildsrud O, Bohlin J, Scheffer L, Eldholm V. Rapid scoring of genes in microbial pan-genome-wide association studies with Scoary. Genome Biol. 2016;17(1):238. doi:10.1186/s13059-016-1108-8. PubMed PMID: 27887642; PubMed Central PMCID: PMCPMC5124306.

25. Letunic I, Bork P. Interactive tree of life (iTOL) v3: an online tool for the display and annotation of phylogenetic and other trees. Nucleic Acids Res. 2016:44(W1):W242-5. doi:10.1093/nar/gkw290. PubMed PMID: 27095192; PubMed Central PMCID: PMCPMC4987883.

26. Cheng L, Connor TR, Siren J, Aanensen DM, Corander J. Hierarchical and spatially explicit clustering of DNA sequences with BAPS software. Mol Biol Evol. 2013:30(5):1224-8, doi:10.1093/molbev/mst028. PubMed PMID: 23408797; PubMed Central PMCID: PMCPMC3670731.

27. Croucher NJ, Page AJ, Connor TR, Delaney AJ, Keane JA, Bentley SD, et al. Rapid phylogenetic analysis of large samples of recombinant bacterial whole genome sequences using Gubbins. Nucleic Acids Res. 2015;43(3):e15.

28. Drummond AJ, Rambaut A. BEAST: Bayesian evolutionary analysis by sampling trees. BMC Evol Biol. 2007;7:214. doi:10.1186/1471-2148-7-214. PubMed PMID: 17996036, PubMed Central PMCID: PMC2247476.

29. Minin VN, Suchard MA. Fast, accurate and simulation-free stochastic mapping. Philos Trans R Soc Lond B Biol Sci. 2008;363(1512):3985-95. doi:10. 1098/rstb.2008.0176. PubMed PMID: 18852111; PubMed Central PMCID: PMCPMC2607419.

30. Jombart T, Ahmed I. adegenet 1.3-1: new tools for the analysis of genomewide SNP data. Bioinformatics. 2011:27(21):3070-1. doi:10.1093/ bioinformatics/btr521. PubMed PMID: 21926124; PubMed Central PMCID: PMCPMC3198581.

31. Jombart T, Eggo RM, Dodd PJ, Balloux F. Reconstructing disease outbreaks from genetic data: a graph approach. Heredity (Edinb). 2011;106(2):383-90 doi:10.1038/hdy.2010.78. PubMed PMID: 20551981; PubMed Central PMCID: PMCPMC3183872

32. Inouye M, Dashnow H, Raven LA, Schultz MB, Pope BJ, Tomita T, et al. SRST2: Rapid genomic surveillance for public health and hospital microbiology labs. Genome Med. 2014;6(11):90. doi:10.1186/s13073-014-0090-6. PubMed PMID: 25422674; PubMed Central PMCID: PMCPMC4237778.

33. Antipov D, Hartwick N, Shen M, Raiko M, Lapidus A, Pevzner PA. plasmidSPAdes: assembling plasmids from whole genome sequencing data. Bioinformatics. 2016:32(22):3380-7. doi:10.1093/bioinformatics/btw493.

34. Raven KE, Reuter S, Gouliouris T, Reynolds R, Russell JE, Brown NM, et al. Genome-based characterization of hospital-adapted Enterococcus faecalis lineages. Nat Microbiol. 2016;1(3). doi: 10.1038/nmicrobiol.2015.33. PubMed PMID: 27213049; PubMed Central PMCID: PMCPMC4872833.

35. Howden BP, McEvoy CR, Allen DL, Chua K, Gao W, Harrison PF, et al. Evolution of multidrug resistance during Staphylococcus aureus infection involves mutation of the essential two component regulator WalKR. PLOS Pathog. 2011;7(11):e1002359. doi:10.1371/journal.ppat.1002359. PubMed PMID: 22102812: PubMed Central PMCID: PMCPMC3213104.
36. Bashir A, Attie O, Sullivan M, Sebra R, Singh KV, Altman D, et al. Genomic confirmation of vancomycin-resistant Enterococcus transmission from deceased donor to liver transplant recipient. PLoS One. 2017;12(3):e0170449. doi:10.1371/journal.pone.0170449.

37. Choudhury T, Singh KV, Sillanpaa J, Nallapareddy SR, Murray BE. Importance of two Enterococcus faecium loci encoding Gls-like proteins for in vitro bile salts stress response and virulence. J Infect Dis. 2011;203(8):1147-54. doi:10. 1093/infdis/jiq160. PubMed PMID: 21451003; PubMed Central PMCID: PMCPMC3107556.

38. Taur $Y$, Pamer EG. The intestinal microbiota and susceptibility to infection in immunocompromised patients. Curr Opin Infect Dis. 2013;26(4):332-7. doi: 10.1097/QCO.0b013e3283630dd3. PubMed PMID: 23806896; PubMed Central PMCID: PMCPMC4485384.

39. Top J, Willems R, Bonten M. Emergence of CC17 Enterococcus faecium: from commensal to hospital-adapted pathogen. FEMS Immunol Med Microbiol. 2008;52(3):297-308. doi:10.1111/j.1574-695X.2008.00383.x.

\section{Submit your next manuscript to BioMed Central and we will help you at every step:}

- We accept pre-submission inquiries

- Our selector tool helps you to find the most relevant journal

- We provide round the clock customer support

- Convenient online submission

- Thorough peer review

- Inclusion in PubMed and all major indexing services

- Maximum visibility for your research

Submit your manuscript at www.biomedcentral.com/submit
) Biomed Central 